\title{
Comparison of Choriocapillary Flow Density Between Fellow eyes of Polypoidal Choroidal Vasculopathy and Neovascular Age-Related Macular Degeneration
}

\author{
Mingyue Luo \\ PUMCH \\ Mingzhen Yuan \\ Peking Union Medical College Hospital \\ Jingyuan Yang \\ Peking Union Medical College Hospital \\ Nan Zhao \\ Peking Union Medical College Hospital \\ Rongping Dai \\ Peking Union Medical College Hospital \\ Youxin Chen ( $\nabla$ chenyouxinpumch@163.com ) \\ Peking Union Medical College Hospital
}

\section{Research article}

Keywords: polypoidal choroidal vasculopathy, age-related macular degeneration, optical coherence tomography angiography, contralateral eye, choriocapillary flow density

Posted Date: January 20th, 2020

DOI: https://doi.org/10.21203/rs.2.21264/v1

License: (a) This work is licensed under a Creative Commons Attribution 4.0 International License. Read Full License

Version of Record: A version of this preprint was published at BMC Ophthalmology on April 22nd, 2020. See the published version at https://doi.org/10.1186/s12886-020-01386-0. 


\section{Abstract}

\section{Background}

To compare the choriocapillary flow density (CFD) among the contralateral eyes of polypoidal choroidal vasculopathy (PCV), neovascular age-related macular degeneration (nAMD) and healthy controls with spectral-domain optical coherence angiography tomography (SD-OCTA).

Methods

This is a cross-sectional study. 36 eyes of 36 PCV patients, 35 eyes of nAMD patients and 34 control eyes were included. All sujects underwent the HD Angio Retina $6.0 \mathrm{~mm}$ scan pattern of OCTA (RTVue XR Avanti AngioVue; Optovue, Inc., Fremont, CA, USA). Circles with radius of 1.00, 1.50 and $3.00 \mathrm{~mm}$ were manually selected in choriocapillaries (CC) slab, and CFD was calculated as the percentage of flow area to the whole selected area as CFD-1.00, 1.50, 3.00 respectively. Multivariate linear regression analysis was performed to evaluate the differences of CFD among the three groups after adjusting for age, gender and the existence of drusen.

Results

Mean CFD-1.00, 1.50, 3.00 of nAMD group were $61.09,62.65$ and 65.67 , significantly lower than PCV group $(65.80,66.75,67.86, P=0.000,0.000,0.022)$ and control $(66.17,66.45,68.08, P=0.000,0.001,0.025)$, while no difference were detected between PCV group and control ( $P=0.507,0.798,0.575)$.

Conclusions

CFD of nAMD fellow eyes were significantly lower than that of PCV and control eyes, while no difference was detected between PCV and control group, indicating different role of CC loss in early pathogenesis of nAMD and PCV.

\section{Background}

Polypoidal choroidal vasculopathy (PCV) is characterized by orange-red nodules in fundus examinations, polypoidal lesions during indocyanine green angiography (ICGA) and tremendous bleeding of posterior pole. It has raised many controversies since first described by Yannuzi in 1980s(1), especially its pathogenesis and whether it's a subtype of neovascular age-related macular degeneration (nAMD), or a distinct disease within pachychoroid disease spectrum(2), due to obvious heterogeneity that lies in their clinical, pathophysiological and epidemiological features and treatment responses to anti-vascular endothelium growth factor (VEGF) agents(3).

The pathogenesis and nature of PCV remains to be elucidated, so is its characteristic lesions, namely polyps and branching vascular network (BVN). In recent years, multi-model imaging technologies allows more precise view of polypoidal lesions and PCV choroid. Specifically, with optical coherence tomography angiography (OCTA), a novel imaging modality reflecting choroid flow with higher accuracy and resolution compared to traditional dye-based ophthalmic angiography(4), emerging evidence suggests the classical 
polypoidal lesions manifest as tangled vessels at the edge of BVN(5). This finding further complicates our knowledge that whether there is actual difference between PCV and nAMD, since the characteristic lesions of PCV and choroidal neovascularization (CNV) in NAMD are essentially both vascularization in nature and there might be actually no "polyps" or "aneurysmal vessels"(6).

Since both PCV and nAMD tend to be bilateral(7), fellow eye study of unilateral-involved patients may shed light on their preclinical alterations. Indeed, non-exudative neovascularization of the fellow eye was found associated with pachychoroid pigment epitheliopathy in PCV and nAMD patients(8), and an increased trend of choriocapillaris nonperfusion was detected in the fellow eyes of nAMD using OCTA (9). Additionally, CNV formation was observed more frequently where retinal pigmented epithelium (RPE) and outer retinal abnormalities were accompanied with pachyvessels in PCV patients(10). It remains to be clarified whether there is a direct causal effect between pachychoroid and PCV pathogenesis, yet RPE alterations seems a secondary downstream event occurring in later stage of the disease. This differs from nAMD, in which the interplay between RPE and choriocapillaries $(\mathrm{CC})(11,12)$ plays a central role in the early phase, and finally elevated VEGF gives rise to CNV.

Choriocapillary flow density (CFD) detected with OCTA has emerged as a potential metrics to evaluate inner choroid vasculature with fast non-invasive imaging, which has been extensively utilized in various retinal diseases (13-16). However, there has not been a comparison between PCV and nAMD in their fellow eyes with OCTA. This study aimed to compare the contralateral eyes of PCV and AMD in terms of CFD at an early stage without signs of neovascularization.

\section{Methods}

This is a cross-sectional study of unilateral PCV and nAMD patients recruited from June 2017 to June 2019 at Peking Union Medical College Hospital. Healthy controls were also included. The study was approved by the institutional review board (S-K631) and was conducted in accordance with the tenets of the Declaration of Helsinki.

PCV was diagnosed based on the presence of focal hyperfluorescent spots and/or BVN lesions on ICGA on ICGA within the first 6 minutes. nAMD was diagnosed based on CNV in fundus fluorescein angiography (FFA) without polypoidal lesions or BVN in ICGA. Two masked ophthalmologists (M.L. and M.Y.) reviewed the early to late phases of the angiography images independently. A third retinal consultant (Y.C.) arbitrated in case of any discrepancy. The inclusion criteria for patients were unilateral PCV or nAMD cases with complete medical records and at least 1 OCTA scan. Patients and controls were excluded if (1) any signs of neovascularization in the included eyes, namely exudation in FFA or ICGA for patients, and BVN or blood flow signal between RPE and Bruch's membrane in OCTA for all; (2) comorbid with other ophthalmic diseases except for myopia less than $-3.0 \mathrm{D}$ and mild cataract; (3) cloudy refractory media impedes examination of the posterior pole (4) OCTA scan quality was lower than 7.

All patients received comprehensive ophthalmologic examinations, including best-corrected visual acuity (BCVA), spectral domain- optical coherence tomography (SD-OCT) and SD-OCTA (RTVue XR Avanti AngioVue; Optovue, Inc., Fremont, CA, USA) and simultaneous fluorescein angiography ICGA (Heidelberg Retina 
Angiography 2, HRA2, Heidelberg Engineering, Heidelberg, Germany). HD Angio Retina $6.0 \mathrm{~mm}$ scan pattern of each subject was acquired and used for further analysis. Circles with radius of $1.00,1.50$ and $3.00 \mathrm{~mm}$ were manually selected in CC slab, and CFD was calculated as the percentage of flow area to the whole selected area, which were generated by the built-in software, as CFD-1.00, 1.50, and 3.00 respectively.

Statistical analyses were performed using SPSS 22 (IBM Corporation, Armonk, NY, USA). Chi square test and Mann-Whitney $U$ test were used for comparing categorical and continuous variables respectively. Fisher's exact test was used if the expected numbers were small. Normally distributed continuous data were presented in mean and standard deviation (SD), otherwise data were presented in median and interquartile range (IQR). Multivariate linear regression analysis of CFD was performed to evaluate the differences in CFD between the contralateral eyes of PCV and nAMD, and healthy eyes after adjusting for age, gender and the existence of drusen. A P value less than 0.05 was considered significant in all aforementioned analysis.

\section{Results}

36 eyes of 36 PCV patients (mean age: $63.3 \pm 8.8$ years), 35 eyes of nAMD patients (mean age: $67.0 \pm$ 8.7 years) and 34 eyes of 17 control (mean age: $60.1 \pm 7.7$ years) were included. Basic demographic and clinical characteristics of the subjects are summarized in Table 1. 
Table 1

Baseline Demographics and Clinical Characteristics of Subjects

\begin{tabular}{|c|c|c|c|c|c|c|}
\hline & $\begin{array}{l}\text { PCV } \\
(N=36)\end{array}$ & $\begin{array}{l}\text { nAMD } \\
(\mathrm{N}=35)\end{array}$ & $\begin{array}{l}\text { Control } \\
(\mathrm{N}=17 \\
\text { 34eyes) }\end{array}$ & $\begin{array}{l}\text { p values } \\
\text { (PCV vs. } \\
\text { AMD) }\end{array}$ & $\begin{array}{l}\text { p values } \\
\text { (PCV vs. } \\
\text { control) }\end{array}$ & $\begin{array}{l}\text { p values } \\
\text { (AMD vs. } \\
\text { control) }\end{array}$ \\
\hline \multicolumn{7}{|l|}{ Demographics } \\
\hline Age, y mean (SD) & $63.3(8.8)$ & $67.0(8.7)$ & $60.1(7.7)$ & 0.091 & 0.059 & $0.001^{a}$ \\
\hline $\begin{array}{l}\text { Gender, female }(\mathrm{N}) \\
(\%)\end{array}$ & $18(50.0)$ & $13(37.1)$ & $10(58.8)$ & 0.275 & 0.459 & 0.071 \\
\hline $\begin{array}{l}\text { BCVA (logMAR) } \\
\text { (IQR) }\end{array}$ & $\begin{array}{l}0.11(0.00- \\
0.10)\end{array}$ & $\begin{array}{l}0.13(0.00- \\
0.22)\end{array}$ & $\begin{array}{l}0.07(0.00- \\
0.10)\end{array}$ & 0.304 & 0.483 & 0.099 \\
\hline \multicolumn{7}{|l|}{ Smoking } \\
\hline $\begin{array}{l}\text { Current/past/non- } \\
\text { smoking }\end{array}$ & $4 / 0 / 32$ & $5 / 3 / 27$ & $3 / 0 / 13$ & $0.096^{\mathrm{a}}$ & $0.374^{\mathrm{a}}$ & $0.127^{a}$ \\
\hline Hypertention (\%) & $4(11.1)$ & $9(25.7)$ & $5(29.4 \%)$ & 0.112 & 0.056 & 0.731 \\
\hline DM (\%) & $4(11.1)$ & $4(11.4)$ & $1(5.9)$ & $0.966^{\mathrm{a}}$ & $0.430^{\mathrm{a}}$ & $0.409^{a}$ \\
\hline $\begin{array}{l}\text { Drusen existence } \\
(\%)\end{array}$ & $8(22.2)$ & $15(42.9)$ & $5(14.7)$ & 0.063 & 0.419 & $0.009 *$ \\
\hline CFD-1.00 (\%) & $\begin{array}{l}65.80 \\
(3.50)\end{array}$ & $\begin{array}{l}61.09 \\
(4.46)\end{array}$ & $\begin{array}{l}66.17 \\
(3.16)\end{array}$ & $0.000^{*}$ & 0.510 & $0.000 *$ \\
\hline CFD-1.50 (\%) & $\begin{array}{l}66.75 \\
(3.32)\end{array}$ & $\begin{array}{l}62.65 \\
(4.13)\end{array}$ & $\begin{array}{l}66.45 \\
(2.71)\end{array}$ & $0.000^{*}$ & 0.888 & $0.000 *$ \\
\hline CFD-3.00 (\%) & $\begin{array}{l}67.86 \\
(2.57)\end{array}$ & $\begin{array}{l}65.67 \\
(3.73)\end{array}$ & $\begin{array}{l}68.08 \\
(2.41)\end{array}$ & $0.009 *$ & 0.716 & $0.005^{\star}$ \\
\hline \multicolumn{7}{|l|}{${ }^{*} \mathrm{p}$ values $<0.05$} \\
\hline
\end{tabular}

All demographic and clinical characteristics for PCV and nAMD patients were matched in terms of age, gender, BCVA, smoking status, hypertension, DM and existence of drusen in the contralateral eyes. The means (SDs) CFD-1.00, -1.50, -3.00 for PCV fellow eyes were 65.80 (3.50), 66.75 (3.32), and 67.86 (2.57) respectively, significantly different from that of nAMD, which were 61.09 (4.46), 62.65 (4.13), and 65.67 (3.73) (P=0.000, $0.000,0.009)$. After adjusted for age, sex and drusen existence, the difference was still statistically significant as shown in Table $2(P=0.000,0.000,0.022)$. No difference was observed for all CFD values before $(P=$ $0.510,0.888,0.716)$ and after adjustment $(P=0.507,0.798,0.575)$ between $P C V$ contralateral and control eyes. While the difference of CFD between nAMD fellow eyes and control eyes was statistically significant before $(P=0.000,0.000,0.005)$ and after adjustment $(P=0.000,0.001,0.025)$. 
Table 2

Multivariate Linear Regression of CFD, Adjusted for Age, Sex and Existence of Drusen

\begin{tabular}{|c|c|c|c|c|c|c|c|c|c|}
\hline & \multirow[b]{2}{*}{ PCV } & \multirow[b]{2}{*}{ AMD } & \multirow[b]{2}{*}{ Control } & \multicolumn{2}{|c|}{ PCV vs. AMD } & \multicolumn{2}{|c|}{ PCV vs. Control } & \multicolumn{2}{|c|}{ AMD vs. Control } \\
\hline & & & & $\begin{array}{l}\beta- \\
\text { coefficient } \\
(\mathrm{SE})^{\mathrm{b}}\end{array}$ & $\begin{array}{l}\mathrm{P} \\
\text { values }\end{array}$ & $\begin{array}{l}\beta- \\
\text { coefficient } \\
(\mathrm{SE})^{\mathrm{b}}\end{array}$ & $\begin{array}{l}\mathrm{P} \\
\text { values }\end{array}$ & $\begin{array}{l}\beta- \\
\text { coefficient } \\
(\mathrm{SE})^{\mathrm{b}}\end{array}$ & $\begin{array}{l}\mathrm{P} \\
\text { values }\end{array}$ \\
\hline $\begin{array}{l}\text { CFD- } \\
1.00 \\
\text { (SD) }\end{array}$ & $\begin{array}{l}65.80 \\
(3.50)\end{array}$ & $\begin{array}{l}61.09 \\
(4.46)\end{array}$ & $\begin{array}{l}66.17 \\
(3.16)\end{array}$ & -0.449 & $0.000 *$ & 0.077 & 0.507 & 0.499 & $0.000 *$ \\
\hline $\begin{array}{l}\text { CFD- } \\
1.50 \\
\text { (SD) }\end{array}$ & $\begin{array}{l}66.75 \\
(3.32)\end{array}$ & $\begin{array}{l}62.65 \\
(4.13)\end{array}$ & $\begin{array}{l}66.45 \\
(2.71)\end{array}$ & -0.422 & $0.000^{*}$ & -0.030 & 0.798 & 0.421 & $0.001^{*}$ \\
\hline $\begin{array}{l}\text { CFD- } \\
3.00 \\
\text { (SD) }\end{array}$ & $\begin{array}{l}67.86 \\
(2.57)\end{array}$ & $\begin{array}{l}65.67 \\
(3.73)\end{array}$ & $\begin{array}{l}68.08 \\
(2.41)\end{array}$ & -0.272 & $0.022^{*}$ & 0.070 & 0.575 & 0.296 & $0.025^{*}$ \\
\hline
\end{tabular}

Drusen existence significantly correlated with CFD-1.00 and -1.50, but not 3.00 in the comparisons among all groups (Table 3 ).

Table 3

The Relationship between Existence of Drusen and CFD in multivariate Linear Regression ${ }^{\mathrm{a}}$

\begin{tabular}{|lllllll|}
\hline \multicolumn{3}{|c}{ PCV vs. AMD } & \multicolumn{2}{l|}{ PCV vs. Control } & \multicolumn{2}{l|}{ AMD vs. Control } \\
\hline & $\begin{array}{l}\text { Correlation } \\
\text { Coefficient }\end{array}$ & P & $\begin{array}{l}\text { Correlation } \\
\text { Coefficient }\end{array}$ & P & $\begin{array}{l}\text { Correlation } \\
\text { Coefficient }\end{array}$ & P \\
\hline CFD-1.00 & -0.353 & $0.001^{*}$ & -0.398 & $0.001^{*}$ & -0.348 & $0.001^{*}$ \\
\hline CFD-1.50 & -0.319 & $0.004^{*}$ & -0.386 & $0.002^{*}$ & -0.326 & $0.004^{*}$ \\
\hline CFD-3.00 & -0.110 & 0.062 & -0.234 & 0.068 & -0.172 & 0.159 \\
\hline a Multivariate linear regression of CFD, adjusted for age, sex and existence of drusen. \\
\hline * P values < 0.05
\end{tabular}

\section{Discussion}

In our study, we evaluated the CFD of contralateral eyes of nAMD and PCV with OCTA, and found nAMDcontralateral eyes had significantly lower CFD compared with PCV and control eyes, while the difference wasn't statistically significant between PCV group and control. Although CFD was found negatively correlates with aging(17), it is unlikely that aging itself can account for the difference, since PCV and AMD group were age-matched. Another important factor is the existence of drusen, which is considered a hallmark of dry AMD. 
In this study, nAMD group had higher rate of drusen existence (42.9\%) in the fellow eyes, but the difference didn't reach a significant threshold compared with PCV group $(22.2 \%, P=0.063)$. Besides, drusen existence was found negatively correlated with CFD in areas with a radius of 1.00 and $1.50 \mathrm{~mm}$, but not $3.00 \mathrm{~mm}$, indicating drusen existence primarily associated with decreased CFD of sub-foveal area, not the entire macula. Our finding is consistent with a previous study that vascular density was inversely associated with sub-RPE deposit density(18). Although there is a myriad of overlap between PCV and nAMD, the difference of CFD in their contralateral eyes may serve as a proof of their heterogeneity.

The interplay of RPE and CC loss has always been a hotspot in the pathogenesis of AMD, but their sequential order remains controversial. In various pathological studies, CC lost is proven to precede RPE degeneration and drusen formation $(19,20)$ and with OCTA, Nassisi et al found that CFD predicts the development and enlargement of drusen(21). In addition, lower choroidal perfusion detected with laser Doppler flowmetry was a risk factor for developing CNV in the fellow eyes of nAMD patients(12), and apparent neovascular buds were detected adjacent to areas of CC loss in histopathological study(20). These findings reinforce the role of CC loss in the pathogenesis of in both dry and neovascular AMD. On the other hand, VEGF secreted by RPE is vital for the maintenance of $\mathrm{CC}(22)$, and abnormal RPE morphology and function extends the area of CC loss in various geographic atrophy studies(23). In a histopathological including both forms of AMD, Mcleod et al (23) found a linear relationship between the loss of RPE and CC in GA, while CC dropout was evident in the absence of RPE, and they proposed the primary insult in GA and nAMD may arise from different levels, namely RPE and CC respectively. Overall, the cause-and-consequence relationship of these two surrogate markers remains a challenge, yet the pathology of AMD, despite wet or dry, lies in the RPE-Bruch's membraneCC complex.

While the CFD of PCV-contralateral eyes wasn't different from control, indicating RPE-Bruch's membrane-CC complex isn't involved in the preclinical stage of PCV and may be a downstream effect secondary to primary pathology. Indeed, PCV is a well-recognized disease within pachychoroid disease spectrum, and engorgement of vortex vein is often observed, with correlating choroidal hyperpermeability(24), also confirmed by the pathology showing atherosclerotic change of choroid vessel wall, massive exudation of fibrin and blood plasma at polypoidal lesion $(25,26)$. Apoptosis of smooth muscle cells and choroidal endothelial cells were also observed(25). Chen et al induced polyp-like structures by ligating vortex veins in cynomolgus monkeys(27) These findings above suggest a role of choroidal hemodynamics in PCV pathogenesis(28).

A plethora of studies investigated the difference of choroidal morphology between PCV and nAMD. Subfoveal choroidal thickness (SFCT) is often used as an index, but it fluctuates with circadian rhythm and correlates with age and refractive errors(29). Bakthavatsalam et al(29) found that choroidal vascular index (CVI) of nAMD was lower than that in PCV (64.94 vs 62.54), but the difference wasn't statistically significant ( $P=$ 0.10). Pachyvessels especially diffuse pattern were observed more commonly in thick-choroid PCV(30), and vascular area of typical PCV was significantly larger than $\mathrm{AMD}(31)$. All aforementioned results indicate the two diseases are heterogeneous in terms of choroidal morphology.

Our study had the limitations that are inherent as a retrospective cross-sectional study. Besides, DM, hypertension and smoking status was collected from patients' past medical records which were recorded in a self-report manner, thus not accurate enough to be included into multivariate analysis. Nevertheless, to the 
best of our knowledge, this is the first study that provided quantitative analysis of choriocapillaries among the fellow eyes of PCV, nAMD, and healthy eyes. Our study may provide evidence for the heterogeneity of nAMD and PCV.

\section{Conclusions}

In conclusion, our findings suggest that CFD of nAMD fellow eyes were significantly lower than that of PCV and control eyes, while no difference was detected between PCV and control group, indicating a different role CC loss plays in the early pathogenesis of nAMD and PCV.

\section{Abbreviations}

Polypoidal choroidal vasculopathy, PCV

indocyanine green angiography, ICGA

neovascular age-related macular degeneration, nAMD

vascular endothelium growth factor, VEGF

branching vascular network, BVN

optical coherence tomography angiography, OCTA

choroidal neovascularization, CNV

retinal pigmented epithelium, RPE

choriocapillaries, CC

Choriocapillary flow density, CFD

fundus fluorescein angiography, FFA

best-corrected visual acuity, BCVA

spectral domain- optical coherence tomography, SD-OCT

standard deviation, SD

interquartile range, IQR

\section{Declarations}

\section{Ethics approval and consent to participate}


The study was approved by the institutional review board of Peking Union Medical College Hospital (S-K631) and was conducted in accordance with the tenets of the Declaration of Helsinki. A copy of the written consent is available for review by the Editor-in-Chief of this journal.

No informed consent was obtained from patients in this study since only patient records and past examinations that were accessible to medical professionals were collected for this study in a non-identifiable manner.

\section{Consent for publication}

Not applicable.

\section{Availability of data and materials}

Not applicable.

\section{Competing interests}

The authors declare that they have no conflict of interest.

\section{Funding}

This work was supported by The Non-profit Central Research Institute Fund of Chinese Academy of Medical Sciences (2018PT32029). All authors have no relevant financial relationships to disclose.

\section{Authors' contributions}

ML carried out the entire procedure including the collection of medical records, image evaluation, statistical analysis, drafting the manuscript and manuscript revision. MY and JY contributed to data collection and manuscript revision. $\mathbf{N Z}$ and $\mathbf{R D}$ revised statistical analysis and assisted with language. YC conceived of the study, coordinated and participated in the entire process of drafting and revised the manuscript. All authors read and approved the final manuscript.

\section{Acknowledgements}

Not applicable.

\section{References}

1. Yannuzzi, L.A., et al., Idiopathic polypoidal choroidal vasculopathy (IPCV). Retina, 1990. 10(1): p. 1-8.

2. Balaratnasingam, C., et al., Polypoidal Choroidal Vasculopathy: A Distinct Disease or Manifestation of Many? Retina, 2016. 36(1): p. 1-8.

3. Laude, A., et al., Polypoidal choroidal vasculopathy and neovascular age-related macular degeneration: same or different disease? Prog Retin Eye Res, 2010. 29(1): p. 19-29. 
4. Jia, Y., et al., Quantitative optical coherence tomography angiography of vascular abnormalities in the living human eye. Proc Natl Acad Sci U S A, 2015. 112(18): p. E2395-402.

5. Bo, Q., et al., Appearance of Polypoidal Lesions in Patients With Polypoidal Choroidal Vasculopathy Using Swept-Source Optical Coherence Tomographic Angiography. JAMA Ophthalmol, 2019.

6. Dansingani, K.K., et al., Understanding aneurysmal type 1 neovascularization (polypoidal choroidal vasculopathy): a lesson in the taxonomy of 'expanded spectra' - a review. Clin Exp Ophthalmol, 2018. 46(2): p. 189-200.

7. Kim, Y.T., et al., Development of polypoidal choroidal vasculopathy in unaffected fellow eyes. Br J Ophthalmol, 2012. 96(9): p. 1217-21.

8. Yanagi, Y., et al., Prevalence and Risk Factors for Nonexudative Neovascularization in Fellow Eyes of Patients With Unilateral Age-Related Macular Degeneration and Polypoidal Choroidal Vasculopathy. Invest Ophthalmol Vis Sci, 2017. 58(9): p. 3488-3495.

9. Treister, A.D., et al., Prevalence of Subclinical CNV and Choriocapillaris Nonperfusion in Fellow Eyes of Unilateral Exudative AMD on OCT Angiography. Transl Vis Sci Technol, 2018. 7(5): p. 19.

10. Baek, J., et al., Polypoidal Choroidal Vasculopathy: Outer Retinal and Choroidal Changes and Neovascularization Development in the Fellow Eye. Invest Ophthalmol Vis Sci, 2019. 60(2): p. 590-598.

11. Alagorie, A.R., et al., Quantitative Assessment of Choriocapillaris Flow Deficits in Eyes with Advanced Age-Related Macular Degeneration Versus Healthy Eyes. Am J Ophthalmol, 2019.

12. Boltz, A., et al., Choroidal blood flow and progression of age-related macular degeneration in the fellow eye in patients with unilateral choroidal neovascularization. Invest Ophthalmol Vis Sci, 2010. 51(8): p. 4220-5.

13. Arya, M., et al., Choriocapillaris changes in dry age-related macular degeneration and geographic atrophy: a review. Eye Vis (Lond), 2018. 5: p. 22.

14. Borrelli, E., et al., Topographic Analysis of the Choriocapillaris in Intermediate Age-related Macular Degeneration. Am J Ophthalmol, 2018. 196: p. 34-43.

15. Yang, J., et al., Optical coherence tomography angiography analysis of the choriocapillary layer in treatment-naive diabetic eyes. Graefes Arch Clin Exp Ophthalmol, 2019. 257(7): p. 1393-1399.

16. Wang, Q., et al., Optical coherence tomography angiography in retinal vein occlusions. Graefes Arch Clin Exp Ophthalmol, 2018. 256(9): p. 1615-1622.

17. Pettenkofer, M., et al., Choriocapillaris Flow Density Negatively Correlates With Advancing Age on Spectral-Domain Optical Coherence Tomography Angiography. Ophthalmic Surg Lasers Imaging Retina, 2019. 50(5): p. 302-308.

18. Mullins, R.F., et al., Choriocapillaris vascular dropout related to density of drusen in human eyes with early age-related macular degeneration. Invest Ophthalmol Vis Sci, 2011. 52(3): p. 1606-12.

19. Biesemeier, A., et al., Choriocapillaris breakdown precedes retinal degeneration in age-related macular degeneration. Neurobiol Aging, 2014. 35(11): p. 2562-2573.

20. Seddon, J.M., et al., Histopathological Insights Into Choroidal Vascular Loss in Clinically Documented Cases of Age-Related Macular Degeneration. JAMA Ophthalmol, 2016. 134(11): p. 1272-1280. 
21. Nassisi, M., et al., Choriocapillaris flow impairment predicts the development and enlargement of drusen. Graefes Arch Clin Exp Ophthalmol, 2019.

22. Saint-Geniez, M., et al., An essential role for RPE-derived soluble VEGF in the maintenance of the choriocapillaris. Proc Natl Acad Sci U S A, 2009. 106(44): p. 18751-6.

23. McLeod, D.S., et al., Relationship between RPE and choriocapillaris in age-related macular degeneration. Invest Ophthalmol Vis Sci, 2009. 50(10): p. 4982-91.

24. Chung, S.E., et al., Engorgement of vortex vein and polypoidal choroidal vasculopathy. Retina, 2013. 33(4): p. 834-40.

25. Nakashizuka, H., et al., Clinicopathologic findings in polypoidal choroidal vasculopathy. Invest Ophthalmol Vis Sci, 2008. 49(11): p. 4729-37.

26. Okubo, A., et al., Clinicopathological correlation of polypoidal choroidal vasculopathy revealed by ultrastructural study. Br J Ophthalmol, 2002. 86(10): p. 1093-8.

27. Chen, L.L., et al., Choroid changes in vortex vein-occluded monkeys. Int J Ophthalmol, 2018. 11(10): p. 1588-1593.

28. Ng, D.S., et al., Classification of Exudative Age-Related Macular Degeneration With Pachyvessels on En Face Swept-Source Optical Coherence Tomography. Invest Ophthalmol Vis Sci, 2017. 58(2): p. 10541062.

29. Bakthavatsalam, M., et al., Choroidal structures in polypoidal choroidal vasculopathy, neovascular agerelated maculopathy, and healthy eyes determined by binarization of swept source optical coherence tomographic images. Graefes Arch Clin Exp Ophthalmol, 2017. 255(5): p. 935-943.

30. Baek, J., et al., Morphologic features of large choroidal vessel layer: age-related macular degeneration, polypoidal choroidal vasculopathy, and central serous chorioretinopathy. Graefe's Archive for Clinical and Experimental Ophthalmology, 2018. 256(12): p. 2309-2317.

31. Gupta, P., et al., DETAILED CHARACTERIZATION OF CHOROIDAL MORPHOLOGIC AND VASCULAR FEATURES IN AGE-RELATED MACULAR DEGENERATION AND POLYPOIDAL CHOROIDAL VASCULOPATHY. Retina, 2017. 37(12): p. 2269-2280. 